pendant longtemps : les traces qui en témoi-

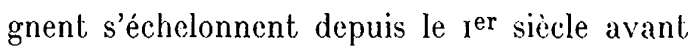
notre ìre (lessons modelés sans tour et lissés, monnaie d'EPAD) jusqu'à la fin du Ive siècle (monnaie d'Arcadius). Pendant ce temps il a été l'objet de réparalions (objets découverts sous le pavage de la construction reclangulaire).

Henri Verdier.

\title{
Deuxième supplément au Recueil des bronzes de Bavai
}

La publication du Recueil des Bronzes de Bavai ${ }^{1}$ el le premier supplèment du Recueil, paru ici-même ${ }^{2}$, ont incilé les auteurs et plusieurs conservaleurs de musées à poursuivre le repérage des bronzes figurés de provenance bavaisienne. Un certain nombre de statuettes que l'on croyait perdues onl été retrouvées dans les dépôts mentionnés dans le Recueil. Ainsi, grâce à ses patientes recherches, M. M.-F. Mariën, conservaleur adjoint aux Musées Royaux d'Art et d'Histoire de Bruxelles, a pu mettre la main sur la plupart des bronzes égarés au cours des aménagements successifs de ces musées el, spécialement, de la section de Belgique ancienne ${ }^{3}$. D'autre part, Mre Christiane Piérard, conservatrice de la Bibliothèque publique de Mons, a pu retrouver un bronze bavaisien conservé au Musée archéologique de la ville ${ }^{4}$.

En plus des statuettes et fragments de bronzes figurés, recueillis récemment au cours

gne, autant qu'on en puisse juger d'après sa répartition, paraît avoir été principalement agricole (Gabriel FourNIER, Essai sur le peuplemenl de la Basse Auvergne ì l'époque gallo-romaine, dans la Revue d'Auvergne, t. 73, 1959, p. 129-161). On comprend donc mal cerlains vestiges obscrvés sur des hauteurs d'accès incommode et peu propres à la culture, telles que, par exemple, le puy d'Isson (ou de Solignat), Nonette, le puy SaintAndrí, Lsson (G. et P.-F. FoukNisk, Relevé des slalions à polerie hallstatienne découverles en Basse Auvergne, dans le Bull. hist. el scienl. de l'Auvergne, t. I.XIII, 1943, p. 101-108, nos 20, 29, 30, 31). Peut-être ces vestiges seraient-ils ceux d'anciens lieux de culte?

(1) G. Fatder-Frytmans, Recueil des bronzes de Bavai, VIII e supplément à Gallia, 1957 (Sigle : Recueil).

(2) G. Falder-Frytmans et Henri Biével.et ; Supplement au Recueil des Bronzes de Bavai, dans Gallia XVI (1958), p. 243-251.

(3) Recueil, p. 32 et note 39.

(1) Recueil, nos 187 et 188. des fouilles qui se poursuivent à Bavai, un certain nombre de pièces, conservées en divers dépôts, avait échappé à l'auteur du Recueil; elles se devaient de figurer dans ce supplément. Ajoutons que quelques bronzes ont été altribués par erreur au site bavaisien et que la découverte par M. Guillouet, conservateur du Musée de Douai, de l'inventaire, daté de $1834^{5}$ des pièces cédées à cette ville par l'abbé Carlier, curé de Bavai, apporte au problème bien des données nouvelles. Ces faits mérilaient d'être signalés, d'autant plus qu'ils permettront de cerner avec plus de précision quelques types de pièces issus d'ateliers bavaisiens ${ }^{6}$.

$1^{\circ}$ Bronzes découverls au cours des fouilles de Bavai depuis 1958.

1. Mars (pl. I; . - - Dépôt de fouilles de Bavai, no 8 z. 131. Découvert le 28 août 1958 , entre le mur qui borde, vers l'angle N-E., le portique oriental et l'enceinte du Bas-Empire, à $1 \mathrm{~m}$. 90 sous le niveau de la place voisine, dans une couche de cendres. H. $6 \mathrm{~cm} .2$. Manquent l'avant-bras gauche, la lance, la majeure partie du bouclier.

Fonte pleine, patine vert noir. Le dieu est, représenté nu, debout, appuyé sur la jambe gauche, la droile étant portée en arrière. Le bras droit est levé à hauteur de l'épaule. L'avant-bras forme avec lui un angle droit. La main repliée maintenait une lance. Le bras gauche, plié, légèrement écarté, maintient le bouclier appuyé sur l'épaule relevée. Un casque a cimier coiffe la chevelure bouclée et couvre la tête jusqu'à la nuque.

(5) Recueil, p. 21-26 et, spécialement, p. 24.

(6) G. Faider-Feytmans, Bronzes figurés issus d'aleliers bavaisiens, à paraître dans la Revue Archéologique. 
Inédit.

Cette statuette se rattache à une série connue qui ne comporte pas moins de huit pièces déjà repérées (Recueil, nos 7 à 14).

2. Doigt (pl. I). — Dépôt de fouilles de Bavai no 8 z. 117. Découvert le 28 août 1958 dans le même site que le Mars no 8 z. 131. L. $6 \mathrm{~cm} .7$; diam. $2 \mathrm{~cm}$. 5. Brisé au-delà de l'articulation unissant les deux premières phalanges.

Fonte pleine, patine sombre avec lavelures vertes. Extrémité de l'index, probablement gauche, d'une statue masculine, légèrement hypermétrique. La troisième phalange est un peu ployée. L'ongle est écrasé en haut et à droite.

\section{Inédit}

3. Pied (pl. I). - Dépôt de fouilles de Bavai, $\mathrm{n}^{\circ}$ z. 94. Découvert le 20 août 1958 dans le même sile que les deux pièces précédentes, mais un peu plus à l'ouest. L. $2 \mathrm{~cm} .3$; larg. $1 \mathrm{~cm}$. 2. Brisé au coup de pied. Manque de talon.

Fonte pleine, patine noire. Partic antérieure du pied gauche d'une statuette peut-être féminine, étant donné la délicatesse de la facture.

Inédit.

$2^{0}$ Bronzes de provenance bavaisienne découverts en diffërents dépôts.

4. Fragment de statue (pl. I). -- Dépôt de fouilles de Bavai, $\mathrm{n}^{0} \mathrm{z} 4172$ bis. Découvert le 17 octobre 1947, au cours du déblaiement du sous-sol de la cella centrale, à l'ouest du portique occidental dans le même site que le fragment de draperie z. 4172 (Recueil, no 121). L. $18 \mathrm{~cm}, 8$; larg. $9 \mathrm{~cm}, 3$. Forte oxydation, dépôt calcareux, surtout au revers.

Fragment moulé, patine verte. Présente plusieurs côtes; l'extrémité de deux d'entre elles recouvre celle des autres. Ne s'agirait-il pas de la partic supérieure d'une aile de victoire?

Inédit.

5. Cheval avec cavalier (pl. 11). - Musćes royaux d'Art et d'Histoire, Bruxelles, no 1013 - P. 23. Acquis en 1856. Dépôt antéricur : collection Arthur Dinaux, de Valenciennes.
Découvert en $1813^{7}$. L. $4 \mathrm{~cm}, 5 ;$ h. $4 \mathrm{~cm}, 3$. Les bras du cavalier sont brisés.

Fonte pleine, patine noirâtre. Le cheval, les oreilles dressées, la crinière esquissée, est représenté au galop, les pattes de devant arquées, les pattes de derrière lendues. Le cavalier, nu, a les jambes tendues et appliquées au haut des pattes antéricures du cheval qu'il semble guider ainsi. La têle du cavalier à profil bovin n'a rien d'humain. Une abondante crinière lui couvre la tête et lui pend dans le dos. Des cercles ocellés creux la constellent ainsi que la queuc et les pattes du cheval.

Cette pièce peut être utilement comparée à des chevaux amulettes découverts dans le nord de la lirance, telle la figurine de bronze de Marcheville (Somme) ${ }^{8}$ qui remonterait à la période de Ia Tène.

Inédil.

6. Fragment de plaque métroaque (pl. II). Musées royaux d'Art et d'Histoire, Bruxelles. B. 1723. Acquis en 1912, faisait partie d'un lot de 47 fragments divers et de deux busles de bronze (voir ci-après nos 16 et 17 - Recueil, nos 190-191) provenant de Bavai. Donné dans l'inventaire comme poignée de coffret ou de meuble déformée. Dépôt antérieur : coll. Carpentier - Attenelle, à Roisin ${ }^{9}$. II. 8 centimètres, larg. $10 \mathrm{~cm}, 4$. Seuls le centre et la partic droite de la plaque ajourée sont conservés mais l'extrémité est repliée. Toute la pièce, très oxydée, semble avoir été soumise à l'action d'un feu très violent.

(7) Des douze bronzes de la collection Arthur Dinaux, cédés au musée bruxellois, un seul, une poignée de porte avec buste de silène (Recueil, no 214) avait pu être repéré en 1957. Grâce aux recherches poursuivies par M. M.-E. Mariën, dix d'entre eux ont été retrouvés (voir infra, nos 11 a 15 et 17), auxquels s'ajoutent une fibule en forme de tigre et trois statuettes de caractère exotique qui ne provenaient certainement pas de Bavai (Recueil, nos 101 à 103). Le cheval au cavalier, Suppl. II, no 5 , ainsi que les serpes volives nos 7 et 8 provenant de la collection Dinaux ont été repérés, en plus, dans les réserves des Musées Royaux d'Art et d'llistoire, toujours, grâce aux recherches persévérantes de M. M.-F. Mariën. Manque encore l'amulette phallique (Kecueil, n 240 ) qui déjà n'avail pu être retrouvée en 1944 .

(8) Baron de Lö̈, Calalogue ... de la Belgique ancienne, Musées royaux d'Arl el d'Histoire, II, Les âges du mélal, 1931, p. 170, fig. 77, 2

(9) Rerueil, p. 37, n. 39. 


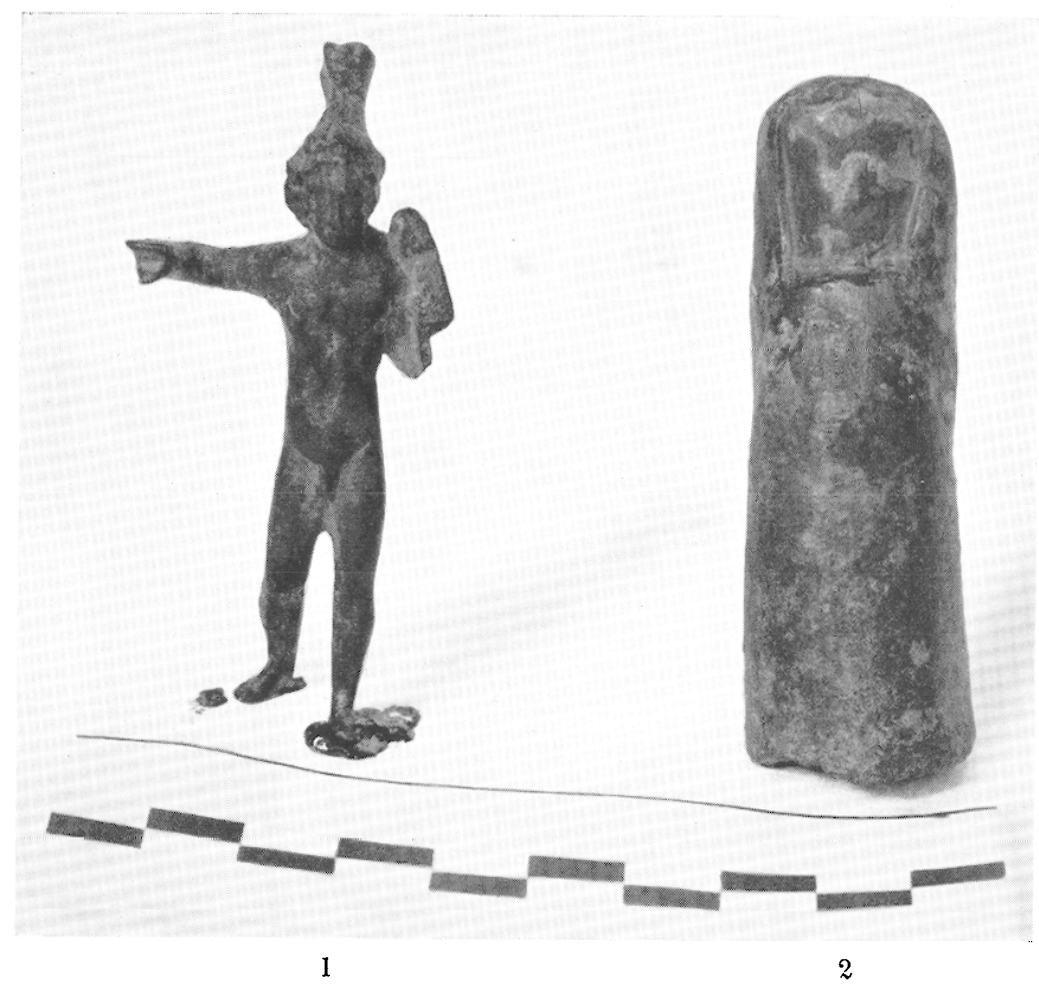

Pianche I.

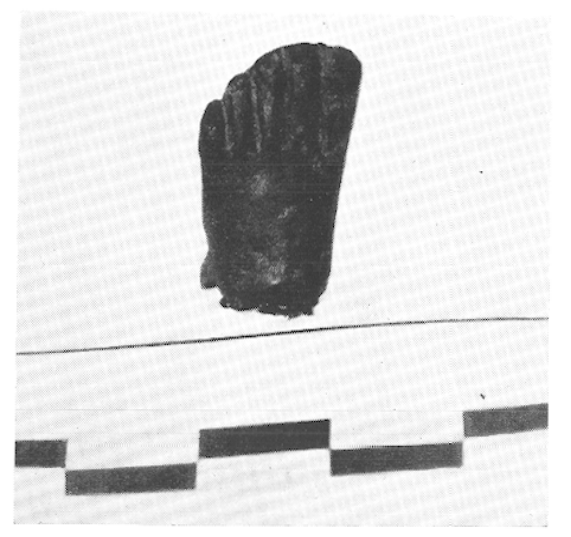

3

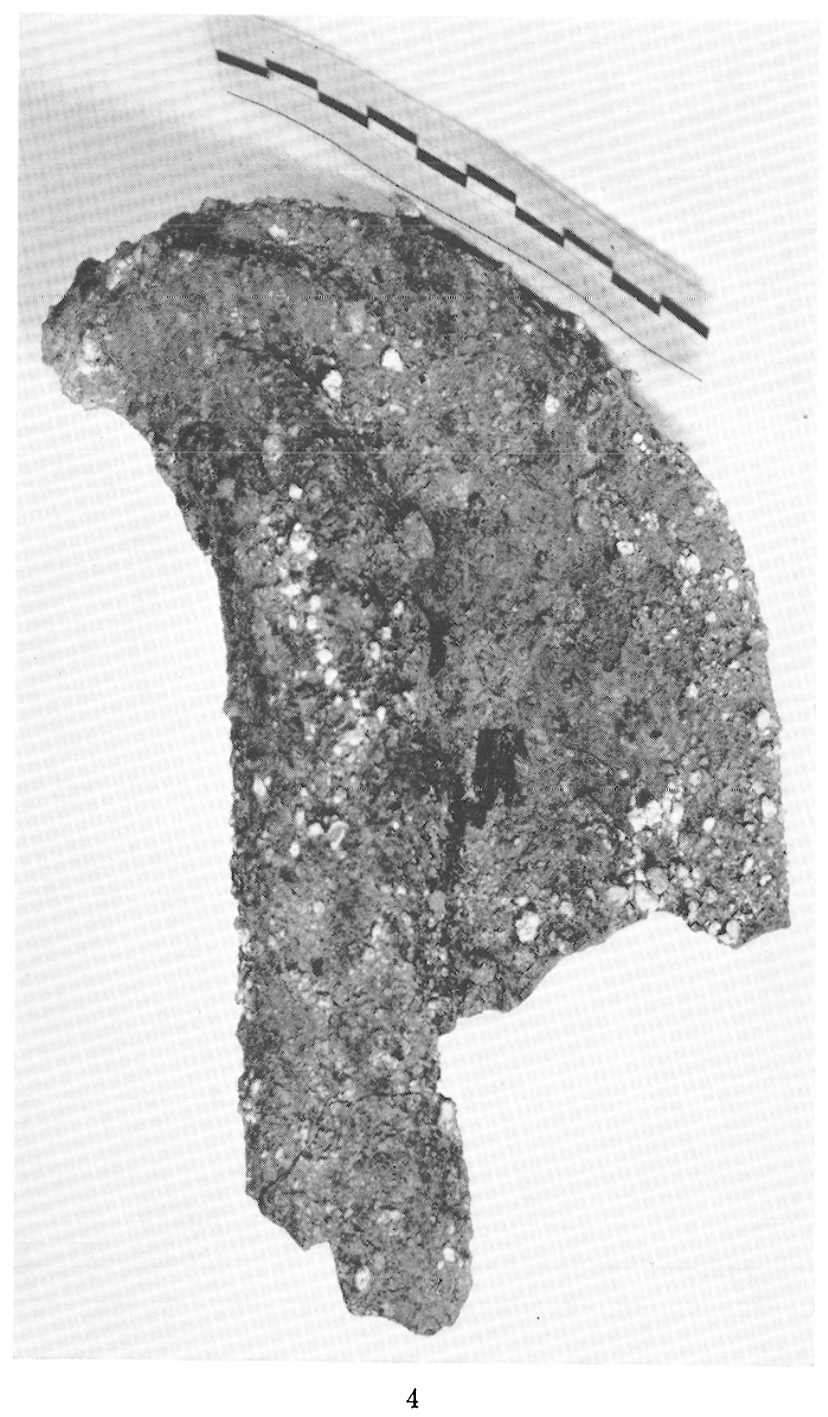


Bronze coulé, patine verte. Au centre se reconnaît le buste de Cybc̀le et un fragment de la couronne tourelée qui la coiffe. Le drapé de l'encolure est encore visible. Le lion dont les pattes postérieures sont appuyées sur l'épaule gauche de la déesse est encore distinct. Toutefois le mufle est complètement écrasé, seule une partie de la crinière faite de traits creux parallèles est décelable. Au revers de la pièce, tordu, le buste d'Attis, coiffé, sur un rang de boucles, du bonnet phrygien très déformé. Le visage est épaufré. Le buste enté sur des pétales est fixé sur une pomme de pin renversée, dont le relief est évoqué par des traits transversaux formant des losanges au centre desquels se trouve un petit cercle creux.

Ce fragment de plaque, dont la facture rappelle celle de la plaque découverte à Bavai en 1951 (Recueil, no 196, pl. XXXV), s'ajoute à une série déjà importante recueillie à Bavai même ou le long des routes qui en rayonnent. V. Recueil, no 195 (bibliographie).

Inédit.

7 et 8 . Serpes votives (pl. III). - Musées royaux d'Art et d'Histoire, Bruxelles, 1025 DD 63. Acquises en 1856. Antérieurement, collection Arthur Dinaux, de Valenciennes. H. 7,2 et $7 \mathrm{~cm}$. 4. Partie inférieure brisée.

Bronze coulé. Patine verte. La première serpe est large, plate et en croissant ; l'emmanchement est simulé par deux godrons. La deuxième, mince et réduite, se termine par une tige dont la base est de plan quadrangulaire. Le manche simulé est rond et terminé de part et d'autre par deux godrons.

\section{Inédit}

$3^{0}$ Bronzes de provenance bavaisienne signalés dans le Recueil et retrouvés dans leurs dépôts respeclifs.

9. Mercure (pl. III). - Musées royaux d'Art et d'Histoire, Bruxelles, 1011 B 245 (z. 13). Acquis en 1856. Dépôt antéricur : Collection Arthur Dinaux (Recueil, $n^{0}$ 100). A fait partie d'un lot de "Trois figurines de bronze représentant des personnages debout \%. Les deux autres figurines, également retrouvées dans les réserves des Musées Royaux ne sont pas galloromaines pas plus que celle provenant de la même collection (Recueil no 103) et donnée dans l'inventaire des Musées Royaux comme "homme nu debout, statuette fragmentée". H. 5 centimètres. Manquent la jambe gauche, le bas de la jambe droile, l'aileron droit et le caducée. Statuette très usée. Visage épaulré.

Fonte pleine, patine noirâtre, profonde oxydation. Le dicu nu, debout, tend une bourse de la main droile.

Cette statuette fait partie d'une série très nombreuse, issue d'ateliers bavaisiens (v. Recueil, nos 18 à 26).

Тн. Justr, Catalogue des collections composant le Musée royal d'antiquilés, d'armures et d'artillerie, Bruxelles, 1864, z. 13.

10. Statuette votive féminine (pl. III). Musées royaux d'art et d'Ilistoire, Bruxelles, no 1009 (z. 11)-B 110. Acquise en 1856. Dépôt antérieur : collection Arthur Iinaux, de Valenciennes. Faisait partie d'un lot donné dans le calalogue Juste comme formé de "quatre petites statuettes en bronze représentant des femmes). Ce lot relrouvé au complet (voir $\mathrm{n}^{\text {os }}$ suivants 13 à 15 ) a fait l'objet d'une note manuscrile du Baron de Loë sur les fiches d'inventaire : "Pièces douteuses, au moins comme provenance ". Cette réticence est sans objet, car la statuette $\mathrm{n}^{\circ} 12$ est celle signalée dans le Recueil sous le no 109 et donnée par l'archilecte Niveleau ${ }^{10}$ comme trouvée à Bavai en 1824 . H. $4 \mathrm{~cm}, 5$. Manquent la tête, la main gauche et l'avant-bras droit.

Fonte pleine. Patine vert sombre. Statuette féminine debout, vêtue d'un chiton talaire maintenu par une ceinture. Un manteau partant de l'épaule gauche passe sous la hanche droite et est ramené sur le bras gauche levé. Statuette très plate. Les plis du chiton sont marqués au revers.

Il s'agit bien de la statuetle dessinće par Niveleau (Recueil, $\mathrm{n}^{\circ}$ 109, pl. $\mathrm{X} \times \mathrm{VI}$ ) et que nous suggeirions comme telle, p. 72. La main droite de la statuette tendait vraisemblablement une patère -- voir $n^{\circ} 11 \ldots$ Dès lors, elle ferait partie, ainsi que les statuettes suivantes, d'un groupe d'images votives populaires

(10) Sur l'activité d'Antoine Niveleau, directeur des fouilles de Bavai, du 20 novembre 1826 au 20 juin 1827 , voir Recueil, p. 31 et 32 . Il laissa en manuscrit un Bavay ancien el moderne (1830), conservé sous les cotes 3799-3800 à la bibliothèque de l'Institut de France. Cité sous le sigle Nrveliau. 
Planche II.

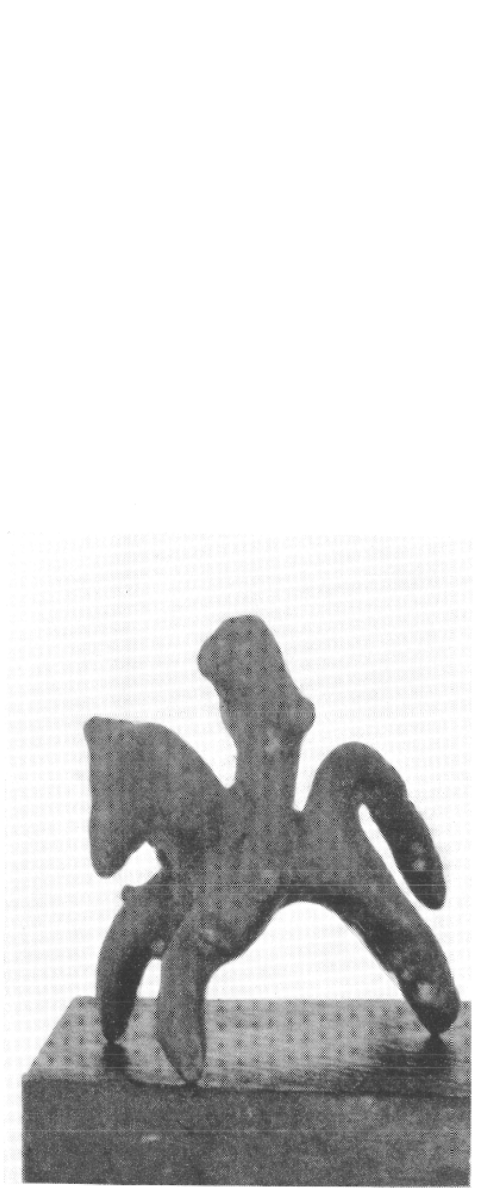

5

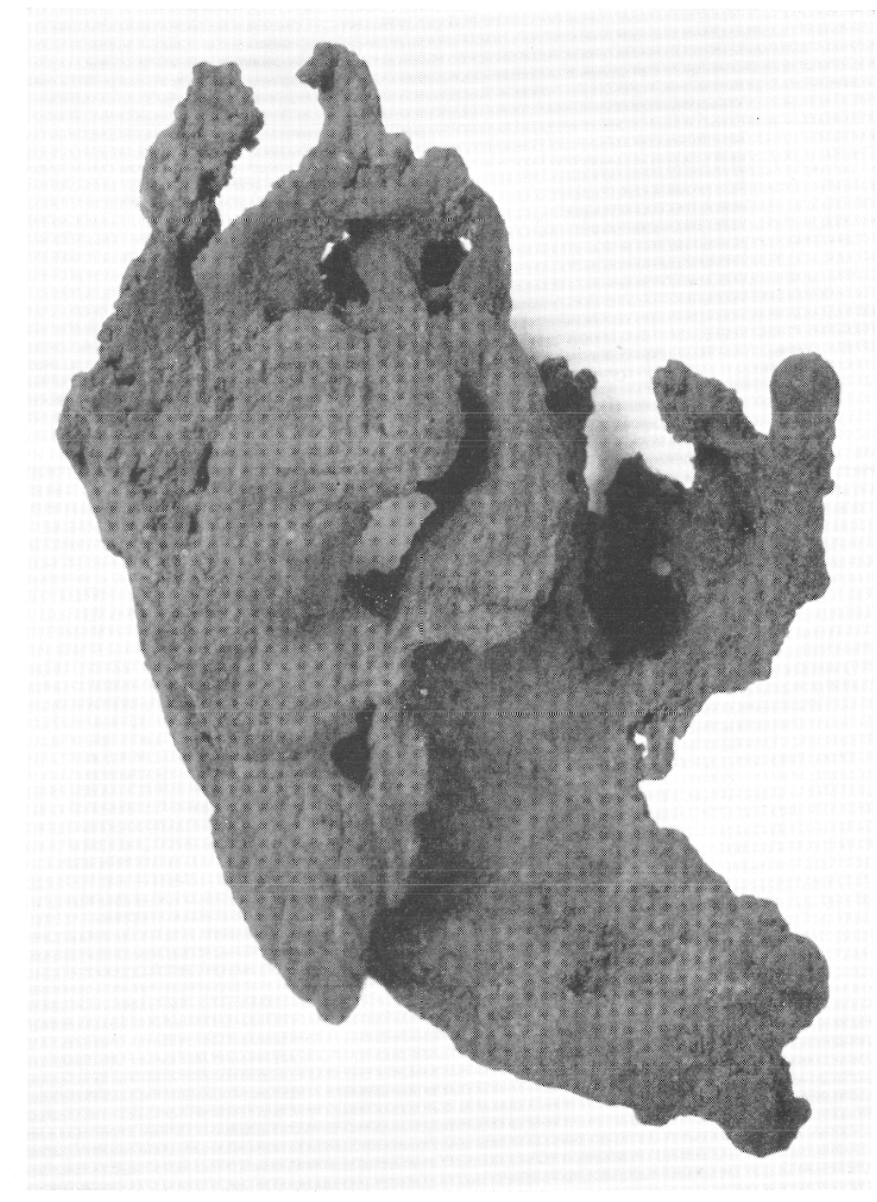

6

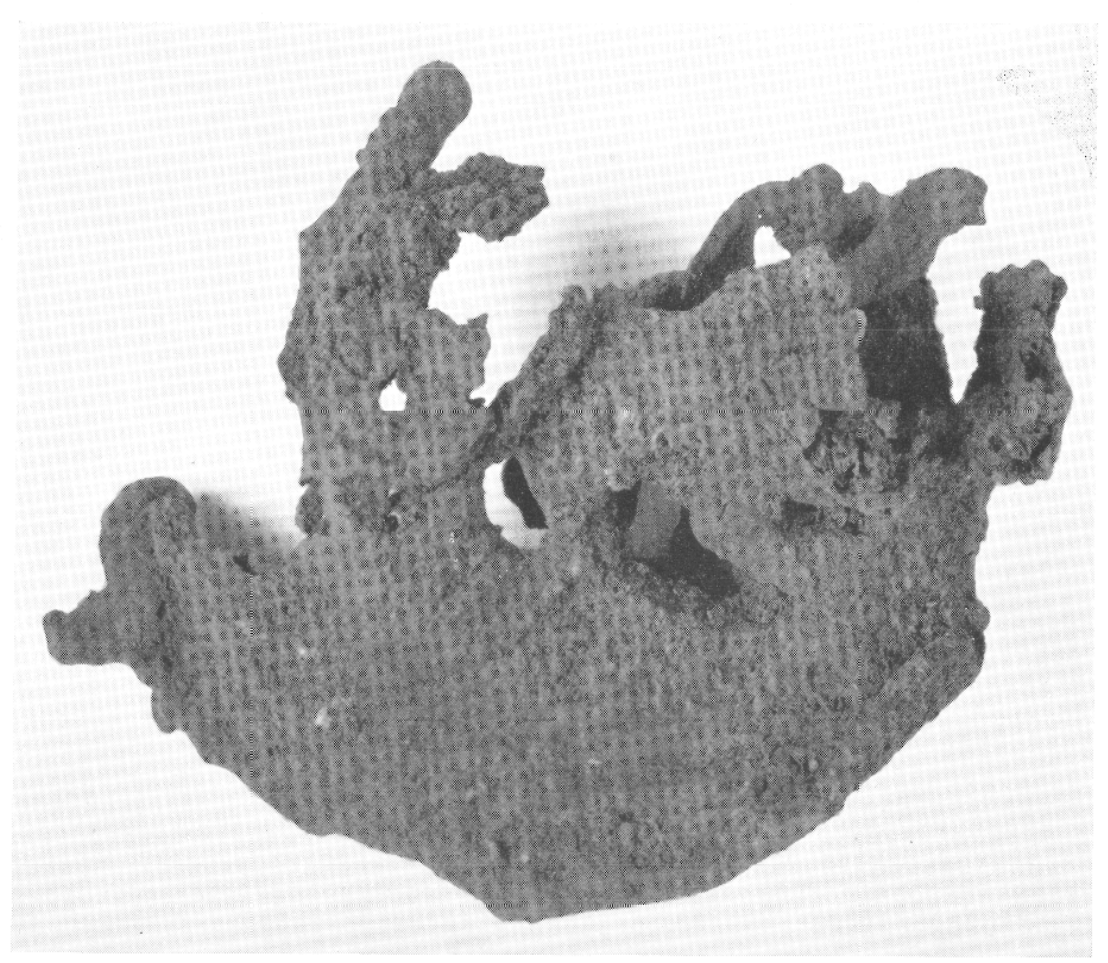

6 
(Malres) peut-être issues d'un atelier bavaisien (Recueil $\left.\mathrm{n}^{\circ \mathrm{s}} 105,106\right)$.

Nivelwal, pp. 80 et 567 , pl. 231 ; Jiste, z. 11 ; Recueil, no 109, pl. XXVI.

11 et 12. Statuettes votives féminines (pl. II I). - Musées royaux d' $\Lambda$ rt et d'histoire, Bruxelles, nos 1009 (311)-B 111 et 112. $\Lambda$ cquises en 1856. Dépôt antérieur : colleclion Arthur Dinaux de Valenciennes. $1^{\circ} \mathrm{H} .7 \mathrm{~cm}, 4$. Manque le bras gauche ; $20 \mathrm{H} .12$ centimètres. manque l'avantbras droit.

Fonte pleine. Patine noirâtre. Femmes debout, vêlues du chiton Lalaire, maintenu à la taille par une ceinture. Un manteau, fixé sur l'épaule gauche, passe sur la hanche droite et retombe sur le bras gauche. La première tend de la main droite une patère. Coiffure à bandeaux ondés terminés par deux mèches pendant dans la nuque. Visages grossièrement esquissés.

Comme la précédente, ces statuettes sont très plates el de facture élémentaire. Il s'agit évidemment de pièces votives de séric.

Justr, Z. 11 ; Recueil, nos 111 et 112.

13. Statuette votive masculine (pl. IV). Musées royaux d'Art et d'Histoire, Bruxelles, $\mathrm{n}^{n} 1009$ (z. 11) B 113. Acquise en 1856. Dépôt antérieur : collection Arthur Dinaux, de Valenciennes. $H$. avec tenon : 9 centimetres. Manque l'avant-bras droit.

Fonte pleine. Patine noire. Personnage masculin, debout, drapé dans un manteau pendant jusqu'aux pieds qui pose sur l'épaule gauche, retombe en pan dans le dos et dégage l'épaule droite et le torse. Les cheveux forment un bourrelet autour de la tête. Le visage est grossièrement esquissé. Sous les pieds, tenon coulé avec la pièce.

Il s'agit bien d'une statuette masculine, contrairement à ce qu'avance l'inventaire (cf. $\left.\mathrm{n}^{\circ} 10\right)$.

Pièce très plate el de facture élémentaire. Il est probable que ce personnage tendait une patère de la main droite (cf. Recueil, nos 89 à 91). Peut-être s'agit-il d'un génie tutélaire.

Juste, Z. 11 ; Recueil, no 113

14. Enfant à l'oiseau (pl. IV). - Musées royaux d'Art et d'Histoire, Bruxelles, no B 94 (487), R 2. Acquis en septembre 1847. Dépôt antérieur : collection Ilector Bochard, de
Bavai11. Aurait été trouvé dans la Sablière Delbauve-Copin. $4.5 \mathrm{~cm}, 2$. Intact, mais oiscau el mains usées. Deux trous ont élé forés sous le siège, pour fixer la pièce sur un socle.

Fonte pleine. Patine vert très foncé. Petit garcon nu assis, les jambes pliées. Il maintient des deux mains, appliqué contre l'épaule gauche, un oiseau aux ailes déployées qui lui mord l'oreille gauche. La têle de l'enfant est couchée sur l'épaule droite. Les traits poupins ont une expression douloureuse. Fossette au menton. La chevelure disposée en grosses mêches hélicoüdales se lermine en petites boucles dans la nuque. Le corps potelé, à nombril et sexe marqués, est joliment modelé. Pièce de qualité.

Charmante staluetle qui s'apparente nettement à l'" Enfant au lapin" (Recueil, no 115, pl. XXVI) provenant de la collection Caylus et conservé au Cabinet des Médailles à Paris.

Jestr, R. 2, p. 164 ; Recueil, no 116.

15. Manche de couteau-Tigre (pl. IV). Musées royaux d'Art et d'Histoire, Bruxelles, no B 246 (1012). Acquis en oclobre 1856 ; dépôt antérieur : collection Arthur Dinaux, de Valenciennes $^{12}$. L. $5 \mathrm{~cm}, 2$. Seuls le fauve et un fragment de la lame de fer sous les palles antéricures sont conservés. Pièce très oxydée. Trace de rouille sous l'arriere-train et au revers de la pièce.

Fonte pleine. Paline verte en partie conservée Le fauve (tigre ou panthère) est représenté les pattes antérieures étendues, comme pressées sur une proie. Le mufle est très abimé. Tout le corps est recouvert d'ocelles simulant le pelage. Des traces d'oxyde de fer, laisscés par la lame qui se repliait contre l'animal formant manche,

(11) Sur llector Bochard, voir Re:ueil p. 33 et 34, n. 39. Deux aulres bronzes vendus par lui aux Musées de Bruxelles, nont pu être retrouvés. Ils étaient déjà égarés en 1944; il s'agit du Bacchus enfant (Recueil $\mathrm{n}^{\circ}$ 44) et du coy (Recueil, no 170).

(12) Cet objet faisait partie d'un lot de "deux tigres couchés, figurines tris frustes". Le deuxième tigre (ou panthère) est une fibule dont l'ardillon a disparu (long. $3 \mathrm{~cm}, 5$ ). L'animal, dont les pattes manquent, a le corps et le mufle couverts de dépressions rondes, destinées à recevoir de l'émail et simulant son pelage. La tête est bien conservée. In collier enserre le col. 


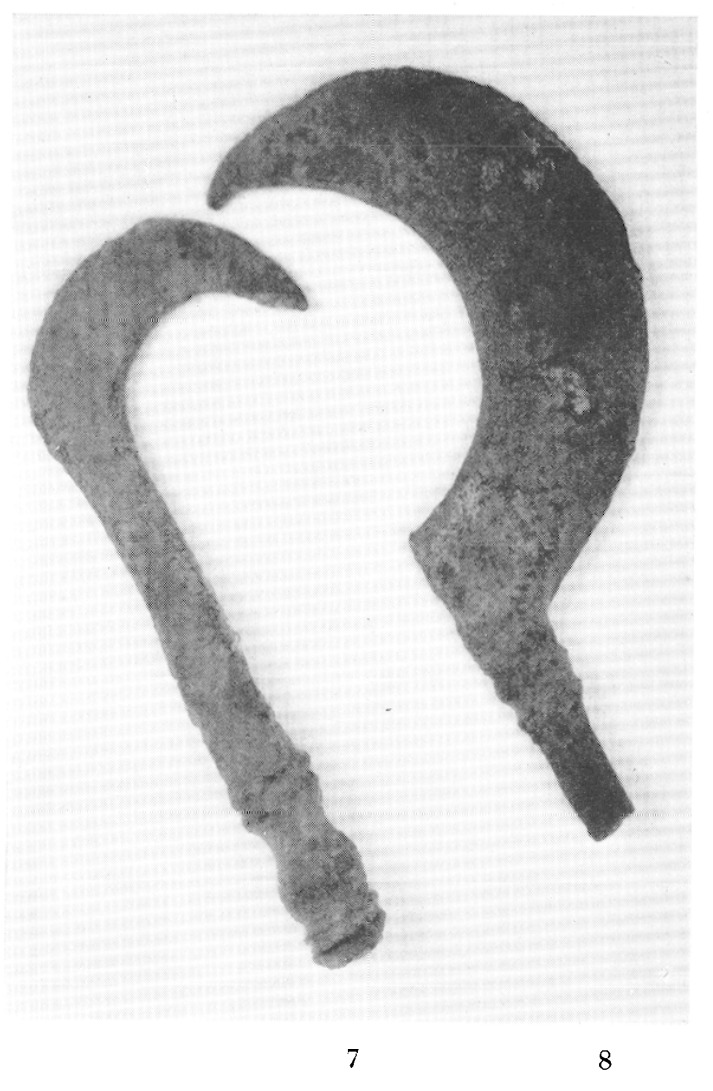

Planche III.

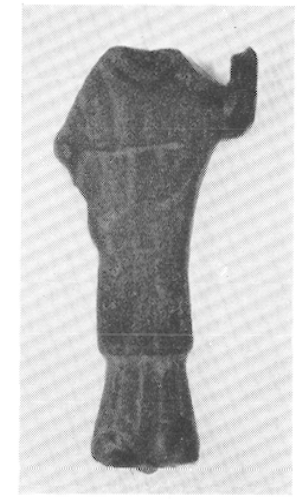

10

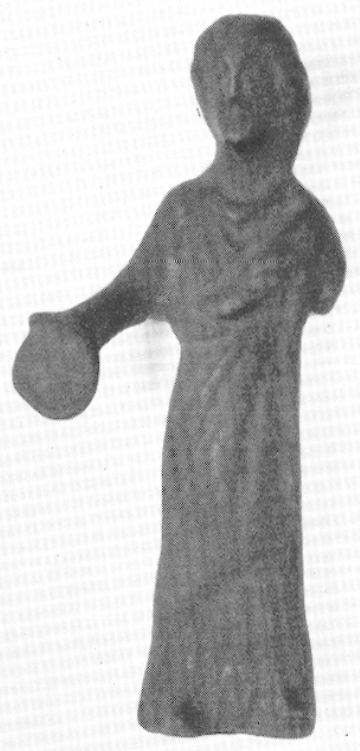

11
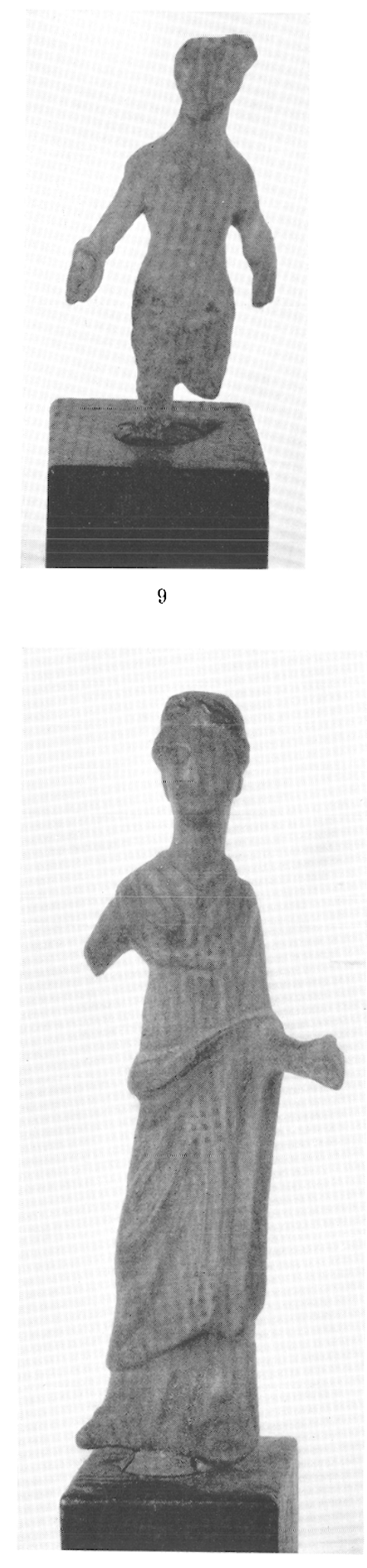

12 
sont visibles tout au long d'un des côtés du fauve.

Un type de couteau de poche replié apparenté a été retrouvé à Roisin (Hainaut) dans les substructions d'une villa (cf. Calalogue ne Lö̈, III, p. 174, fig. 5).

Juste, Z 14, p. 194 ; Recueil, no 134.

16 et 17. Bustes d'Attis (pl. IV). - Muscés royaux d'Art, et d'Histoire, Bruxelles, no 1723. Acquis en octobre 1912. Dépòt antérieur : collection Carpentier-Attenelle, de Roisin. Font partie d'un lot, inventorié sous le seul no 1723 , de 49 objels de bronzes donnés comme non figurés, parmi lesquels se trouvail le fragment de plaque métroaque no 6 . H. $4 \mathrm{~cm}, 7$; larg. $2 \mathrm{~cm}, 8$ et $3 \mathrm{~cm}, 2$. Le deuxicme busle mesurait près de 5 centimètres, mais le haut de la coiflure est tordu. Il est fortement corrodé. Manque un pétale du fleuron près de l'épaule gauche du premier buste.

Fonte pleine, patine noire avec corrosion verte. Buste enté sur cinq pétales. Visage poupin et rond. Bouche pelite, nez épaté, yeux à pupille creuse. Sur les tempes, deux rangs de mêches superposées se rejoignant au sommet du front sous une coiffure effilée. $\Lambda u$ revers du buste, une épaisse mortaise d'l $\mathrm{cm}, 4$, perforée, en bronze. Le deuxième buste, un peu plus large que le premier, est trop corrodé pour permettre une description.

Un buste semblable, egalement pourvu d'une mortaise, été retrouvé à Bavai (Recueil, no 181 , pl. XXXIV. Il appartient au Musce de Lille (no 2453) et faisait partie de la collection Crapez (h. $4 \mathrm{~cm}, 6$ ). D'autre part, Niveleau reproduit, pl. 234, en l'affadissant, une piece semblable ayant appartenu entre 1824 et 1829 à la collection Dessenis, de 13avai. Ces bustes avaient été considérés à lor't comme des personnagres bacchiques. En fait, comme l'a fort justement fait remarquer M. I.ucien Lerat (Revue des Iiludes anciennes, t. I.X (1958), p. 491), il s'auil d'images grossières d'Attis, l'étrange coiffure étant une déformation du bonnet phrygien. Ces appliques sont à rapprocher des bustes d'Attis qui flanquent les plaques mélroaques, mais aucun d'eux ne porte trace de fixation d'une pomme de pin à la base. I'épaisseur des mortaises fait supposer qu'ils étaient fixés à un montant épais, et non à un simple coffret. D'aulre part, ils sont plus larges et plus hauts que les Attis des plaques métroaques.

Recueil, nos 190-191.

18. Peson de balance (pl. IV). -- Musće du Centenaire, Mons (Belgique), inv. no 22. Acquis en 1865. Dépôl antérieur : collection
Nlbert Toilliez. H. 9 cm. 5 . Poids 960 grammes. Pièce très uséc, un fragment manque à la base. Anneau de suspension brisé.

Fonte creuse, remplie de plomb. Patine verte. Poids de balance en forme de buste féminin drapé, de facture très grossière. Le cou est épais, le visage épaufré el lourd. I,a chevelure laurée, séparée en bandeaux par une raie médiane, est retenue dans la nuque en catogan et se termine par deux mèches ondulées, tombant sur les épaules. Au-dessus de la têtte, anneau brisé, perpendiculaire au visage.

Celte pièce est bien celle signalée dans le catalogue de la vente Albert Toilliez, car au revers de son socle de bois se trouve inscrit a l'encre " 22 Toilliez». C'est le numćro qu'elle portait dans le catalogue qui la mentionnait comme "buste avec coiffure surmontée de deux cornes. Bronze trouvé à Bavai " (Calalogue de la collection d'anliquilés classiques, gallo-romaines et franques de feu M. Albert Toilliez, Mons, novembre 1865). D'autre part, J. DE: BムsT, dans Second supplément au recueil d'Anliquilés romaines et gauloises, contenant la descriplion de l'Ancienne ville de Bavai el de Famars, Gand 1813 , p. 40-4l, signale comme trouvée rue du Chătelaire à Bavai " un buste représentant une vieille matrone, ayant un croissant sur la tête. Il est de bronze. H. 3 pouces ". Peut-être s'agit-il du poids conservé à Mons, les fragments de l'anneau brisć ayant èté pris par Toilliez pour deux cornes et par De Bast, s'il s'agit bien de cette mệme pièce, pour un croissant.

Recueil, nos 187-188.

$4^{0}$ Bronzes non bavaisiens.

Trois bronzes ont été donnés, par erreur, comme de provenance bavaisienne dans le Recueil. Il s'agit de trois staluelles de type exceptionnel : "L'Orateur " (Recueil no 96), le Ganymède (Recueil, $\mathrm{n}^{0}$ 65) et l'Applique (Recueil, no 201).

1. Bronze dit l'Orateur, Recueil, no 96 (pl. XXII). Musée de Douai, no 574. Dépôt antérieur: colleclion Carlier à Bavai. Cette tris belle statuette, acquise en 1833, d'une collection bavaisienne a disparu lors de l'incendie du Musée de Douai en 1944. Elle provient en réalité de Neufville-Salesches, près du Quesnoy et a été découverte vers 1780 non loin d'un dépôt monélaire s'échelonnant de SeptimeSévìre à Postume ${ }^{13}$.

2. Ganymède. Recueil, no 65 (pl. XVI). I Iisto-

(13) Voir n. 19. 
Planche IV.

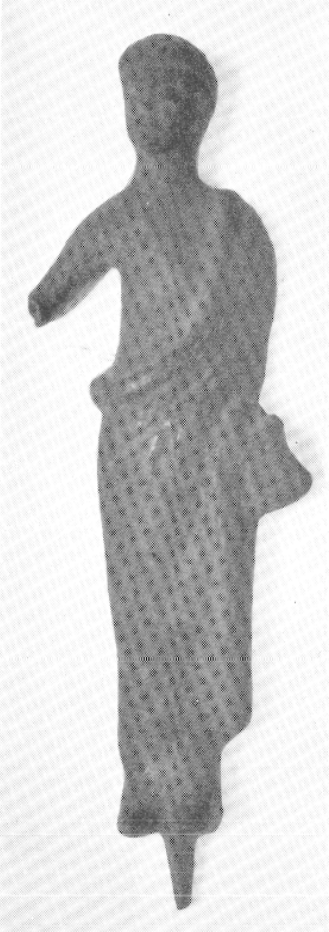

13

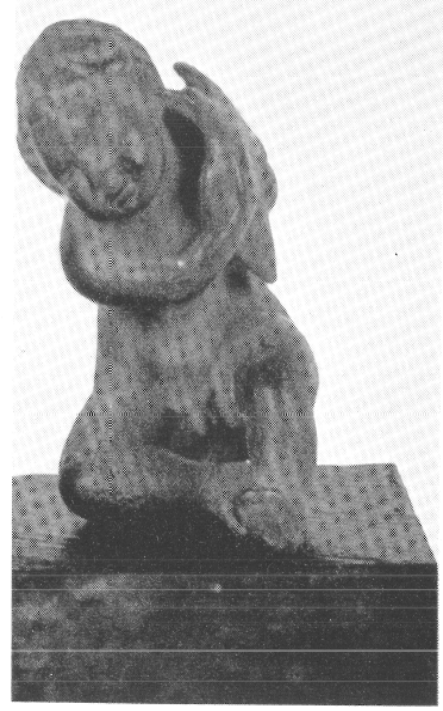

14
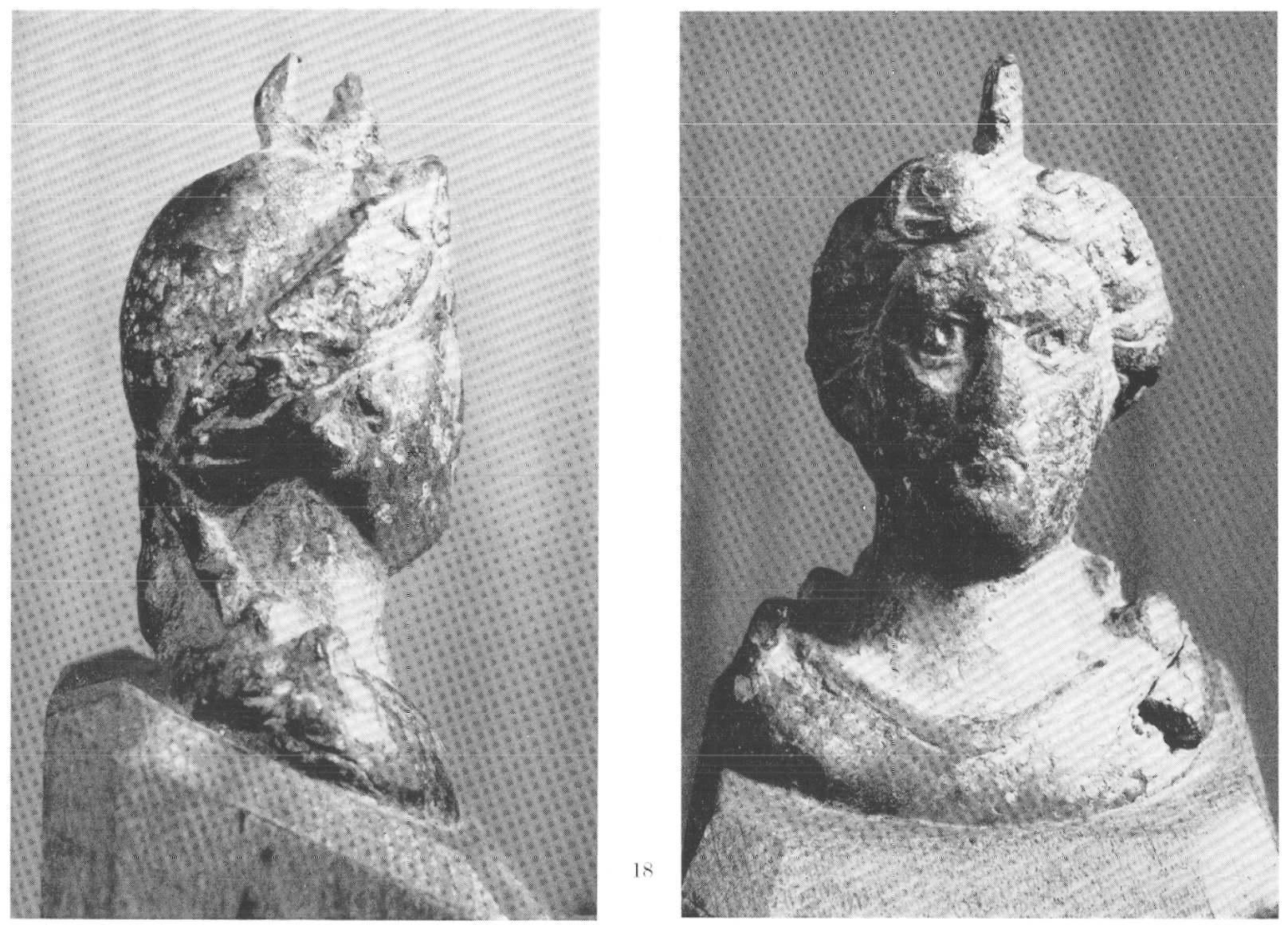
risches Museum (Bâle). Ce bronze fut effectivement découvert en 1803 par l'architecte Nubert Parent non à Bavai, mais à Bâle près du monument qui était considérć alors comme le Grienmatt-Tempel, mais qui semble être le Seplizonium de la ville. En effet, comme l'a signalé très judicieusement $\mathrm{M}^{\mathrm{me}} \mathrm{V}$. von Gonzenbach dans Jahrbuch der Schweizerischen Gesellschaft fur Urgeschichte, 47 (1958-1959). Aubert Parent, avant de devenir en 1813 professeur à l'Académie de Valenciennes ${ }^{14}$ poursuivit, à Bâle des fouilles dont il laissa des rapports précis et d'excellents dessins. C'est durant cette période qu'il recueillit les bronzes dont, par ailleurs, des dessins sont conservés à la bibliothèque municipale de Douai (Manuscrit $n^{\circ} 1037$ ).

3. Applique au géant. Recueil, no 201 (pl. XXXVI). Historisches Museum (Bâle). L'applique qui ne représente pas un silène, mais un géant (L. L.erat, o. c., p. 442) provient de la même fouille d'Aubert Parent et a été trouvée près "des ruines du temple d'Augusta " (Bâle), ce que précise l'inscription qui figure sous le dessin d'Aubert Parent reproduil pl. XXXVI (V. von Gonzenbach, o. c.).

50 Données nouvelles apportées par l'invenlaire des pièces de la collection d'Augustin Carlier, cédées au Musée de Douai.

On le sait : less hériliers de l'abbé Augustin Carlier, curé de Bavai, cédèrenl à la ville de Douai, en 1833, l'importanle collection d'antiques qu'il avait constituée. Comme provenant de cette collection, le Recueil comptait 58 pièces $^{15}$. $\Lambda$ délaut de l'inventaire dressé au moment où la collection était entrée au Musée de Douai, cette supputation avait été établie d'une part d'après les indications qu'avait fournies le conservateur Auguste Cahier, en 1849, dans son Inventaire des Anliquilés ${ }^{\mathbf{1 6}}$, d'autre part et surtout d'aprìs un ensemble de dessins conservés à la Bibliothèque de Douai et

(14) Recueil, p. 26-27 et 31-32.

(15) Aux j̄o pièces énumérées p. 139, sous le mot Carlier, joindre les trois Diane nos 320 , 321 et 321 bis. Cif. ici, la n. 21.

(16) On les retrouve presque toutes dans l'Invenlaire de la section d'archéologie du Musée de Douai, établi par Ed. Delplanque en 1877. qui ont le grand mérite d'être de la main d'Augustin Carlier lui-même ${ }^{17}$.

Or M. Jacques Guillouet, le conservateur du Musée de Douai, a récemment retrouvé, avec des papiers d'Augustin Carlier, un inventaire des pièces de la collection cédées à la ville de Douai. De ce document, établi en 1834, un an environ après la cession, il faut reproduire ici ce qui concerne les bronzes et chercher à résoudre les problèmes qu'il soulève.

Il emplit six pages d'un cahier de sept feuilles de $31 \mathrm{~cm} .3$ sur 22,2 , plices en deux. La première page porte le titre : Invenlaire de la collection d'antiquilés, laissée par l'ancien curé de la ville de Bavai. L'énumération des pièces commence au haut de la p. 3. Elles sont énoncées laconiquement; pas d'autre précision que le prix auquel est évalué, en francs ou en centimes, chaque objet ou groupe d'objets, prix qui ne laisse pas de nous paraitre souvent dérisoire.

$\mathrm{Au}$ bas de la p. 7 on lit ces lignes non signées : Certifié sincère el véritable le présenl inventaire monlant à quatre mille vingt-sept francs cinquante centimes. $\Lambda$ Douai, le 16 juin 1834. C'est prìs de quatre fois plus, $16.000 \mathrm{~F}$., que les héritiers de l'abbé Carlier avaient exigé de la ville de Douai pour lui céder la collection.

Entre le 18 décembre 1828 et le 20 janvier 1829 Valéry Potiez, secrétaire de la Commission Administrative du Musée de Douai, était venu à Bavai pour visiter la collection ; le 20 janvier il présentait sur cette visite à la Commission un rapport qui est transcrit au Registre des Délibérations de cette Commission ouvert le 2 janvier 1828 , fo $23, \mathrm{r}^{\circ}$, à fo $27, \mathrm{v}^{\circ}$. De ce texte il sera utile de rappeler ce qu'il dit des bronzes, en y insérant des numéros qui permettront de Ie comparer facilement avec l'inventaire de 1834, d'une part, celui de 1849 et, le Recueil, d'autre part.

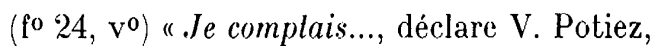
(1 $\left.\begin{array}{lll}1 & \grave{a} & 27\end{array}\right)$ vingl-sept statuettes en bronze, représentant des divinites, Mercure, Apollon, Diane, etc..., plusieurs encore enduites du vernis antique, (28 à 35) huit petits bustes, (36 à 39) quatre petils boucs, (40) un boeuf, (41) un cerf, (42) un sphinx, (43 à 46) qualre coqs, (47) un

(17) Recueil, p. 24. 
sanglier et (48) une têle du même animal... (49) une statuette ailée, (50 et 51) deux tètes de lion, (52) une lête de cigne (Léda), (53) une lête de bélier, (54) un fragment de cet animal, (55) un dauphin, une grappe de raisin entourée de quelques feuilles, (56) un bronze représentanl un amour, (57) une tête de Méduse, (58 ot 59) deux pieds en bronze, (60) une figurine, un pelit panier, (61) une main tenanl une lête d'aigle. $\left(f^{0} 25, v^{0}\right) \ldots$ J'ai remarqué (62) trois soubassemenls de lrépieds, (63 et 64) deux palles de panthère, avec figures, ayant servi de base aux colonnes d'un trépied..."
Un autre des documents récemment retrouvés au Musée de Douai est un cahier formé de trois feuilles de 18 centimètres sur 26 , pliées en deux, et dont les six premières pages contiennent une partie d'un catalogue, établi par l'abbé Carlier, de sa collection; 137 objets y sont inscrils sous des nos allant de 56 à 98 et 101 à 156. Nous reproduirons en appendice ce qui dans ce document - appelons le Catalogue d'Augustin Carlier -- concerne les bronzes ; un chiffre placé ci-dessous dans la colonne de droite, le cas échéant, y renverra le lecteur ${ }^{18}$.

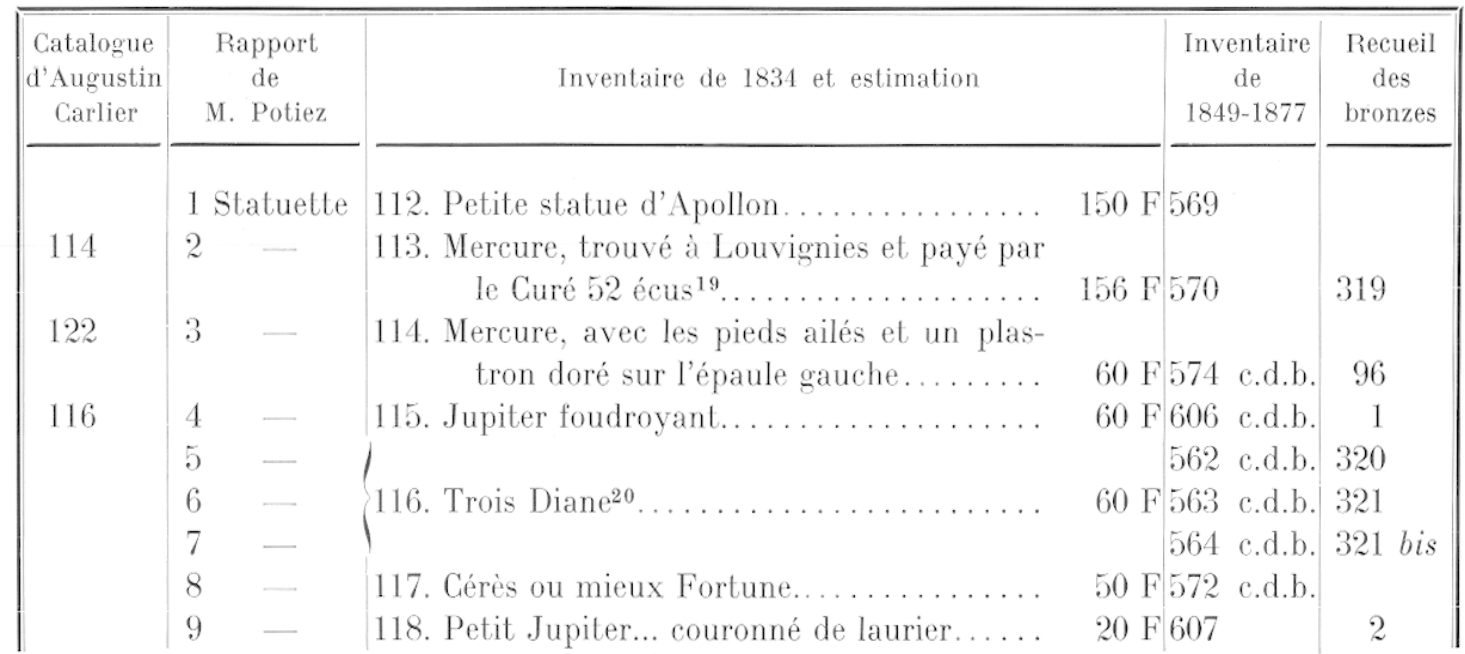

(18) Fin regard de bon nombre d'objets, dans l'inventaire de 1834 , des nos de l'inventaire de 1849 ont été inscrits au crayon par l'un ou l'autre des conservateurs, du moins avec son accord; les conservateurs disposaient peut-être d'indications sur la provenance, qui nous font défaut. I.orsque c'est nous qui proposons de reconnaitre tel objet inventoric en 1834 dans tel objet inventorié en 1849 , le $n^{\circ}$ de l'inventaire de 1849 est accompagné d'un astérisque. Quand $\Lambda$. Cahier indique que telle pièce provient de la collection d' $\Lambda u-$ gustin Carlier, nous faisons suivre le $n^{0}$ correspondant des initiales C (uré) d (e) B (avai); c'est d'ailleurs à elles que recourt souvent $A$. Cahier lui-même pour indiquer cette provenance.

(19) Le bronze no 113 de l'inventaire de 1834, "payé 52 écus par le curé ", donc 156 francs, est le trop cćlèbre Mercure de la Gargotte, un faux. Si le préfet Duplantier l'a détenu quelque temps, dès 1814 , un an après sa découverte, Aug. Carlier était parvenu à faire prévaloir ses droits sur cette pièce, dont d'ailleurs il a reconnu la fausseté. C'est ce Mercure, et non le prétendu Mercure no 114 , qui a "les pieds ailes et un plastron doré sur l'épaule gauche ". Dans la pièce inventoriée sous ce no 114 comme un Mercure, A. Cahier voit un Antinoüs ; e'est le bronze dont Aug. Carlier ecrit dans son catalogue, no 122: Cicero declamans vel alius; au-dessus du mot Cicero se trouve ecrit de la main d'Aug. Carlier le mol Cato. P. 7 du cahier contenant une partie de ce catalogue Aug. Carlier ecrit : " $\Lambda$ Neufville-Salesches, près Le Quesnoi, vers l'an 1780 , un laboureur ... déterra trois cents (médaillesi en argent depuis Septime-Sévère jusqu'aux Postumus. M. le Curé de Bavai les a toutes dans son cabinet, avec une posture d'Apollon déclamant trouvée au mesme lieu ". Sur ces bronzes voir II. Biévelet, Un colleclionneur heureux, Note sur la colleclion d'Augustin Carlier, à paraître dans Ann. du Cercle Arch. de Charleroi. Quant à la "petite statue d'Apollon", no 112, présentement disparue, Aug. Cahier nous en dit : "manquent les mains". Il est possible qu'il y ait confusion aussi pour les prix : ce serait l'orateur qui aurait été estimé non pas 60 francs, mais 150 .

(20) I.e 26 janvier 1815 Aug. Carlier acquérait pour 72 francs des héritiers de feu la veuve de Nicolas Gérin, née Goval, une Diane, "petite figure sur piédestal ", dont elle et son mari lui avaient laissé la jouissance depuis le 4 juillet 1775. Fn 1813, il invite Sébastien Bottin, le secrétaire général de la préfecture du Nord, à venir voir chez lui une Diane récermment entréc dans son cabinet et qu'il soupçonne, elle aussi, de n'être pas antique. Voir l'étude citée n. 19 des trois Diane no 116 re sont pas sur piédestal. 


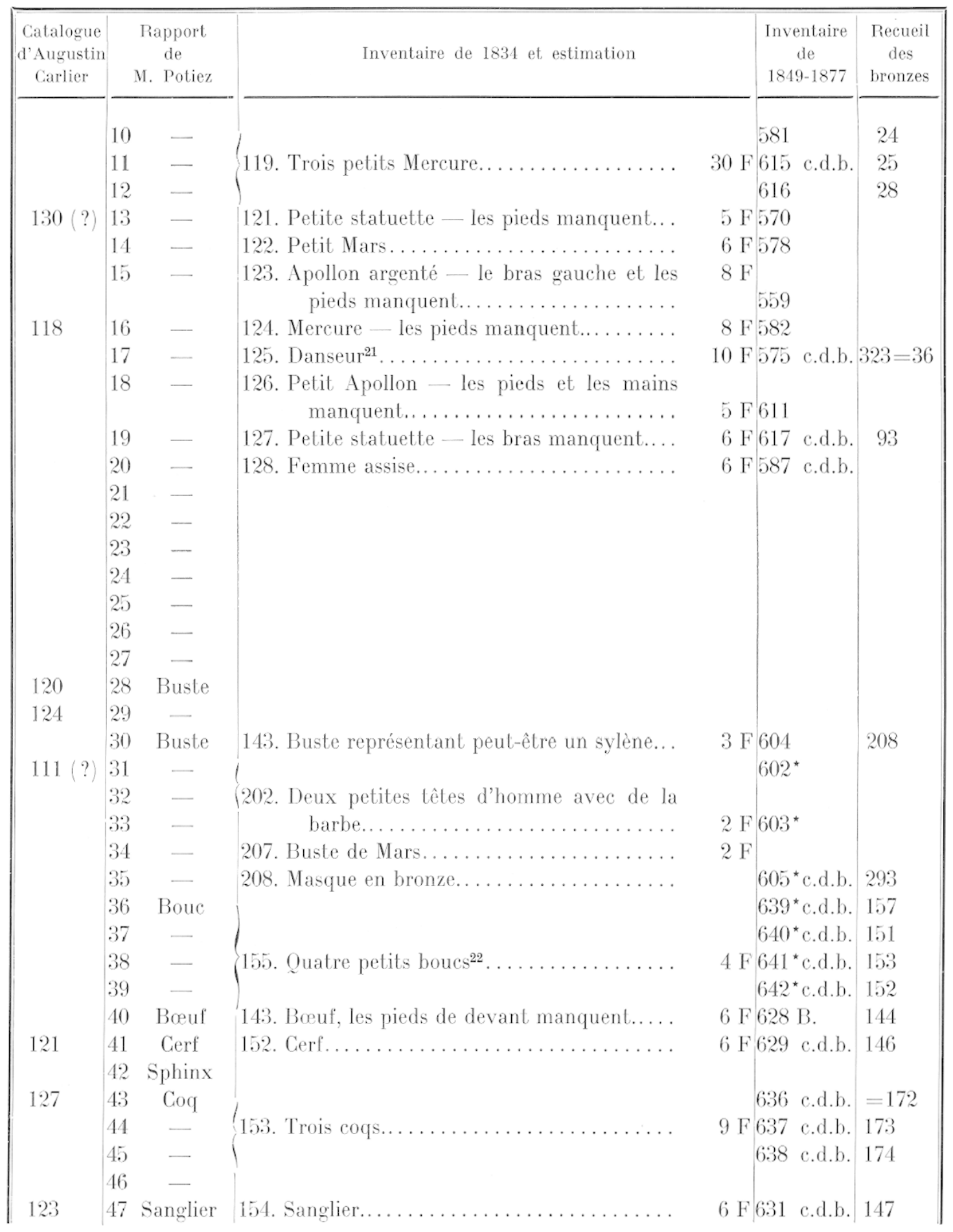

(21) $\mathrm{Si}$ le bronze inventorié en 1834 comme " danseur "est bien la llénade du Recueil no 323 , et un faux moderne, sans doule faut-il ajouter aux bronzes de Bavai présentement disparus un Mercure trouvé en 1812, voir l'clude citée n. 19.

(22) I.es qualre "petits boucs " n 155 de l'inventaire de 1834 sont bien les pieces données comme "chèves" dans l'inventaire de 1849, sous les nos 639, 640, 641, 642. De même la pièce inventorićc aussitôt aprés ces "chèvres", le no 156 de l'inventaire de 1834, en 1349 est inventorièe comme la partie "antérieure d'une chèvre". 


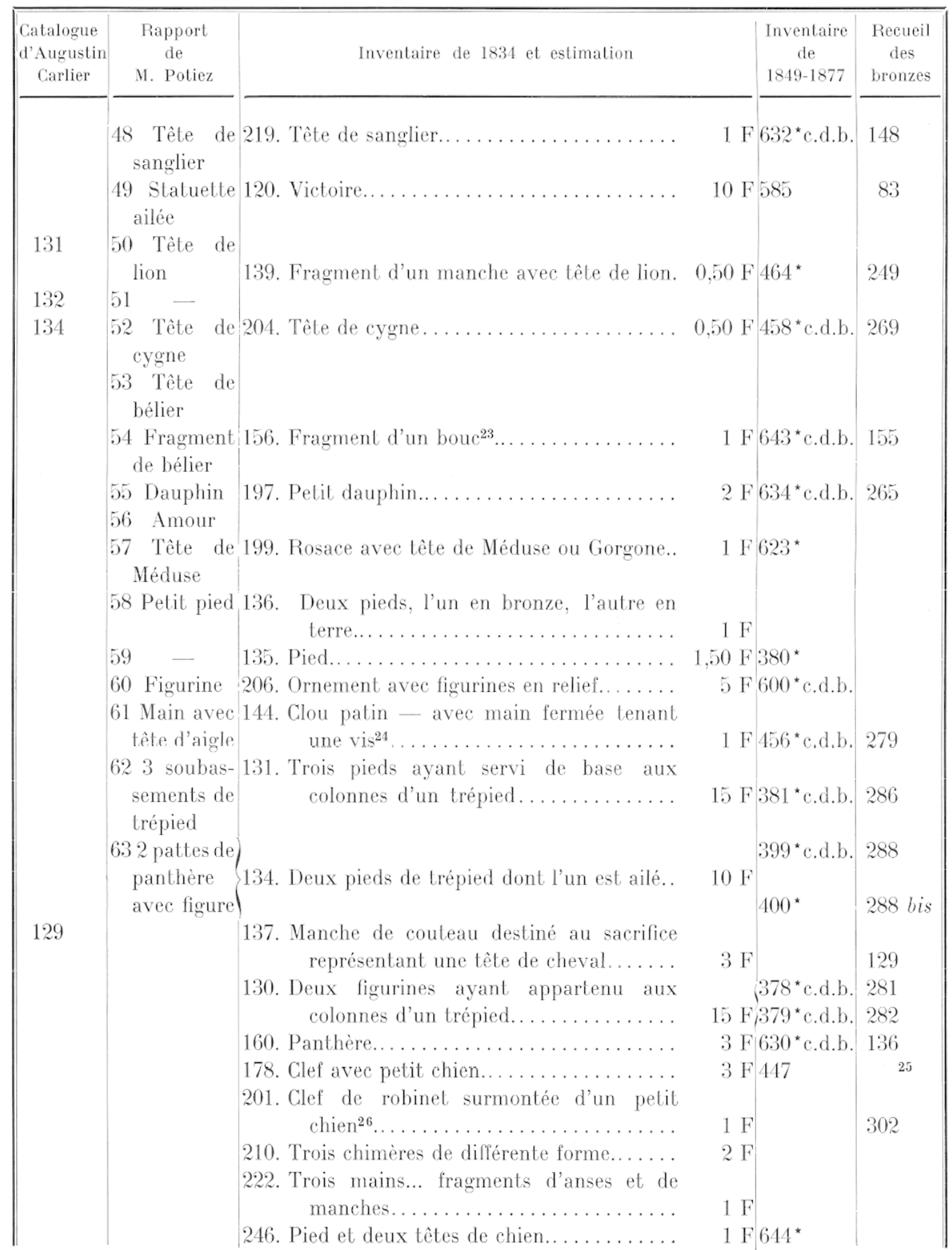

(23) Voir note 22.

(24) V. Potiez parle de "main avec aigle"; dans l'inventaire de 1834 il est question d'un "cloux patin... avec une main fermćc tenant une vis ": peut-ĉlre l'aigle ćtait-il tenu à la main par la vis ; la vis le "main-tenail " et peut-être en 1834 cet aigle avait-il disparu.
(25) Voir dans le Recueil le dernier des alinćas - il est en italiques - où il est question de la " hampe " no 301 .

(26) Comme on peut le remarquer sur le dessin d'Aug. Carlier, ${ }^{\circ} 63, \mathrm{r}^{\circ}$, la paroi de la douille surmontée d'un petit chien est trouce; c'est donc fort vraisemblablement une clef de robinet. 
Du simple rapprochement de ces différentes listes plusieurs constatations se dégagent.

Et d'abord les pièces "remarquées " par V. Potiez, lors de son voyage à Ravai, soit à la fin de 1828 , soit au début de 1829 , ne se trouvent point toutes à Douai en juin 1834 : dans l'entretemps, qui a duré cinq ans et demi, il est vrai, ont disparu sept statuettes sur vingt, trois "pelits bustes " au moins sur huit, un sphinx, un coq, une tête de lion, une tête de bélier et un Amour; ce sont les pièces que nous avons fait précéder dans le texte de V. Potiez des nos $21-27,28,29,31,42,46,51$, $53,56$.

Comment expliquer cette absence? Que sont devenues ces pièces? On ne peut répondre qu'd la $1^{\text {re }}$ de ces questions et seulement par des conjectures; il est possible que les héritiers de l'abbé Augustin Carlier, après le passage de V. Potiez et avant d'aliéner la collection, en aient distrail des objets; il n'est pas moins possible que des pic̀ces se soient égarées ou qu'elles aient été dérobées à l'occasion du transfert de Bavai à Douai, voire à Douai même avant l'inventaire.

Autre fait: V. Potiez ignore plusieurs objets dont fait état l'inventaire de 1834, à savoir :

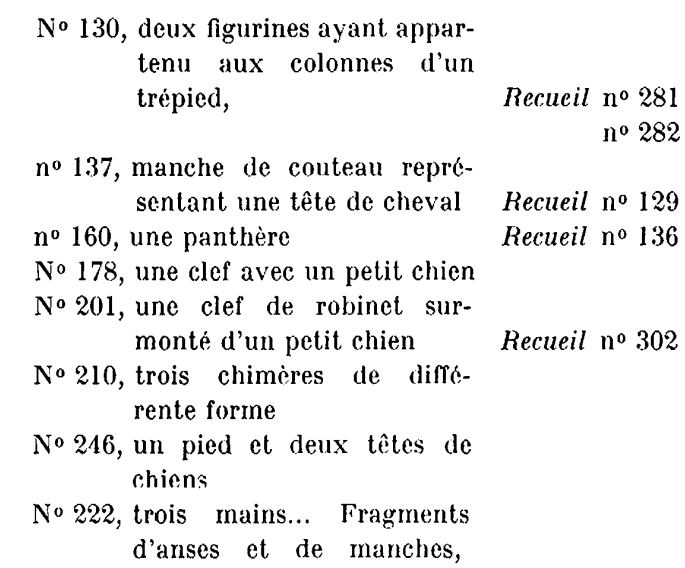

si toutefois il s'agit bien là de bronzes figurés.

V. Potiez est un secrélaire de commission qui sait regarder, évaluer et, d'abord, compter ${ }^{27}$; c'est probablement pour celle raison que ses

(27) Pour les "médailles" V. Potiez ne donne que les résultats de "calculs approximatifs"s : il le dit expressément et s'en explique, Registre des délibérations, fo 26 , $v^{0}$. collègues de la Commission Administrative du Musée l'ont chargé de venir enquêter à Bavai ; il proteste, au début de son rapport, du zèle, de la bonne volonté et du désintéressement qu'il a mis à remplir une mission dont il appréciait l'importance.

Il ne paraît donc pas qu'on puisse expliquer le silence de son rapport sur certaines pièces inventoriées en 1834 en disant que, celles-ci, il ne les a point "remarquées". Mais, si l'on admet que les hériliers de l'abbé Carlier ont pu distraire entre 1829 et 1834 , lelle ou telle pièce, il faut bien admettre qu'ils ont pu tout aussi bien, ne fût-ce que pour compenser leurs soustractions, y réinlroduire tels autres bronzes qu'ils n'avaient point montrés à V. Potiez, la panthère, par exemple, inventoriée en 1834 sous le $n^{0} 160$, sans doute la pièce que De Bast, II suppl., p. 44, avait vue en 1908 .

Entre 1834 et la rédaction de l'Inventaire des Antiquités du Musée de Douai, soit 1849 , disparaissent :

un pied inscrit en 1834 sous le $n^{0} 136$; les trois chimères inscrites sous le $n^{0} 210$; les trois mains (fragments d'anses ou de manches) inscrites sous le $n^{\circ} 222$; un pied inscrit sous le $\mathrm{n}^{\circ} 246$.

Que ces quelques pièces aient pu disparaître d'un musée où elles étaient dûment inventoriées prouverait, s'il le fallait, que d'autres disparitions ont pu se produire précédemment.

Aug. Cahier, dans l'Inventaire des Antiquilés, en 1834, et Ed. Delplanque le copiera dans l'Inventaire de la Section des Antiquités, en 1877, mentionne comme provenant du « Musée de Bavai ")

sous le $n^{\circ} 587$, une prêtresse. Recueil $\mathrm{n}^{0} 107$; sous le $n^{0} 589$, un captif, Recueil $n^{0} 94$; sous le $n^{\circ} 610$, unc têtc de Minerve, Recucil nº 183.

Postérieurement à la rédaction de l'inventaire de 1877, les mots "Curé de Bavai " ont été inscrits au crayon, dans la colonne "provenance", pour le n⿳579, une Vénus pudique, Recueil no 71; pour le no 581, un Mercure, Recueil no 28; pour le n⿳ 588 , une tète de César, Recueil no 86 .

Ces attributions, dont la moitié est très tardive, ne peuvent prévaloir sur le rapport de V. Potiez ou l'inventaire de 1834. Alors que les conservateurs Cahier et Delplanque ont inventorié des objets provenant de la collection 
d'Augustin Carlier, sans mentionner cette provenance, inversement eux ou leurs successeurs ont indiqué cette provenance, s'agissant d'objels qui en réalité ne provenaicnt pas de la collection d'Augustin Carlier.

Enfin pour une série de pièces l'attribution à la collection d'Augustin Carlier repose sur ce seul fait qu'il nous en a laissé des dessins : ce sont

Un Mercure, Inventaires de 1849-1877 $n^{\circ} 566$, Recueil no 30 ;

Un Apollon, Inventaires de 1849-1877 $n^{\circ} 612$, Recueil $n^{\circ} 42$;

Ln fragment de bovidé, Inventaires de 1849-1877, Recueil $n^{\circ} 159$;

Un porcelet, Inventaires de 1849-1877, Recueil $n^{\circ} 150$; Un quadrupède, Inventaires de 1849-1877, Recueil $\mathrm{n}^{\circ} 160$;

Un poisson, Inventaires de 1849-1877, Recueil no 179 ; Un pendentif, Inventaires de 1849-1877, Recueil no 235 ;

In masque d'homme, Inventaires de 1849-1877, Recueil no 329 ;

Lin ornement de suspension, Inventaires de 1849-1877, Recueil $n^{\circ} 307$.

Mais Auguslin Carlier a fort bien pu dessiner des pièces qu'il n'a pas gardées dans sa collection ou des pieces qui n'en ont jamais fait partie.

Ainsi donc l'inventaire de $18: 34$ récemment retrouvé nous l'a fail constater : certains bronzes que, soit sur les dires de Cahier, de Delplanque ou de quelqu'un de leurs successeurs, soit au vu des dessins d'Augustin Carlier, on attribuail à sa collection n'en proviennent pas ; par contre elle a compté plusieurs bronzes dont nous ignorions jusqu'à l'existence; ce sont les pièces qui onl disparu entre 1834 et 1849 : un pied, trois chimères, trois mains, un autre pied, soit, les pièces inscrites dans l'inventaire de 1834 sous les nos $136,210,222$ et 246 .
Elles auraient d'ailleurs été assez modestes, si l'on peut en juger par le prix auquel les évaluait l'inventaire et elles semblent bien, elles aussi, présentement égarées.

\section{H. Biŕvelét et G. Faider-Ffytmans}

\section{Apprandice}

\section{Extrail du Calalogue d'Augustin Carlier}

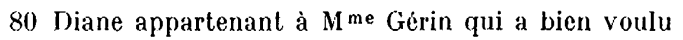
me la céder pendant ma vie.

110 Dauphin, entrée de jet d'eau.

111 Espèce de tête.

112 Cheval en profil.

113 Hure de sanglier.

114 Mercure avec le pétase, forte le dieu des chemins ou chaussées, Vialis.

115 Face imberbe.

116 IIercule ou plutôt Jupiter.

117 Tête de chèvre.

118 Mercure mutilé avec son pétase.

119 Liluus, baton augural.

120 Figure en buste.

121 Cerf.

122 Cicero declamans vel alius (au-dessus du mol Cicero le mot Calol.

123 Sanglier on marcassin.

124 Figure en buste.

125 Pique.

126 IIercule avec la peau de lion et le bout de la massue. 127 Cog de Mercure.

128 Mercure avec le pétase.

129 Tête de cheval, manche d'instrument, manubrium

130 Figure dont les jambes sont cassces.

131 Lion en profil.

132 Lion en profil.

133 Panter (sic).

134 Cou de signe (sic).

135 Figure casquée.

140 Bacchus, figure couronnée de lierre, buste.

142 Oiseau.

143 Petite chèvre.

144 Petit mouton.

184 Botte.

\section{Fouilles de la Trinité, à Callas (Var). Campagne de 1960.}

Lee territoire de la commune de Callas couvre la surface d'une petite dépression que traverse la route nationale 562 de Grasse is Draguignan (fig. 1). Cette dépression est délimilée, au nord par des hauteurs que couvraient encore au siècle dernier des oliviers plantés en "res- tanques" et qu'envahissent maintenant la garrigue et les pins. Au sud-est, une ligne de collines sépare Callas de la commune voisine de Figanières. Seuls d'étroits passages obligés, comme le défilé de la Clue, permetlent à la route de Grasse et à divers chemins secondaires 